\title{
URODYNAMIC STUDIES FOR THE DIAGNOSIS OF LOWER URINARY TRACT SYMPTOMS: EXPERIENCE IN BSMMU
}

\author{
ATM AMAN ULLAH ${ }^{1}$, MD ANAMUL HAQUE ${ }^{1}$, AKM KHURSHIDUL ALAM ${ }^{1}$, AKM ANWARUL ISLAM ${ }^{1}$ \\ ${ }^{1}$ Associate Professor, Department of Urology, Bangabandhu Sheikh Mujib Medical University, Dhaka,
}

\begin{abstract}
Objectives: The aim of urodynamic testing is to obtain objective information regarding urinary bladder storage and voiding function. This investigation provides information of the underlying causes depending on the individual situation and findings.

Materials and Methods: This Retrospective study of the UDS was conducted at Urology department of Bangabandhu Sheikh Mujib Medical University, Dhaka from January 2012 to April 2017. A total of 403 cases of urodynamic studies done. Patient age range was 10-85 years. Female Patient was only 41 and post operative cases was only six. Bladder dairy, Ultrasonography of Kidney, ureter, bladder with maximum cystometric capacity (MCC)and post void residue (PVR), Urine culture were done for most of the patient. Patient with indwelling catheter was remove and clean intermittent catheterization was demonstrated. There was no patient with pace maker or valve replacement. We didn't use routine prophylactic antibiotics, Patients was advice to contact to UDS room physically or over telephone if they feel fever, retention, and other complications. Urodynamic tests include uroflowmetry, postvoid residual measurement, cystometric test, leak point pressure measurement, pressure flow study, and few cases with electromyography.
\end{abstract}

Results: Among them most cases are equivocal and obstructive cases are ranked second about 68 cases (16.87 These were Benign Prostatic Hyperplasia (BPH), stricture urethra, atonic bladder, and dysfunctional voiding. Only five patients had urinary tract infections $(1.2 \%)$ with rise of temperature but only two had cultural positive and treated with sensitive antibiotics. Organism were streptococcus aureus and pseudomonus. One female and one male patient develop retention and relived by continuous catheterisation and after 7 days removed catheter.

Conclusions: Urodynamics could help most diagnosis of LUTSs in most of the cases. We are not yet in using video urodynamics instead urethrocystoscopy may help in assessing the urethra and bladder neck.

Key words: Urodynamics, lower urinary tract, BSMMU.

Bangladesh J. Urol. 2018; 21(1): 8-13

\section{Introduction}

Urodynamic studies are very useful to characteristic features of various types of lower urinary tract symptoms, including frequency, urgency, urinary incontinence, bladder outlet obstruction and neurogenic bladder[1].

Correspondences: ATM Aman Ullah, Associate Professor, Department of Urology, Bangabandhu Sheikh Mujib Medical University, Dhaka,
Urodynamic studies (UDSs) involve the investigation of urinary tract function/dysfunction by various methods $[2,3]$. such as uroflowmetry, filling cystometry (CMG), pressure-flow studies, and assessment of urethral closure [including urethral pressure profilometry and measurement of the leak-point pressure (LPP)]. These tests can be combined with simultaneous electromyography and/or imaging. 3 Video urodynamics 
are the gold standard investigation for patients with lower urinary tract symptoms (LUTSs)[4]. The aim of urodynamic testing is to obtain objective information regarding urinary bladder storage and voiding function. Basic investigations provide information of the underlying causes. Depending on the individual situation and findings, further urodynamic investigations are helpful or indicated. Prior to conservative therapy, a routine urodynamic investigation is not indicated.

The ICl-RS Think Tank recommends that the diagnostic process for patients with LUTD can be redesigned. Carefully delineated and evidence based LUTD syndromes may better indicate, personalize and improve the outcome of initial management, and may also contribute to improved and rational selection of patients for invasive UDS [5]. Urodynamic investigations should be performed preferably preoperatively, especially in case of overactive bladder symptoms, prior incontinence surgery, or disordered bladder emptying. The assessment of urethral function should be considered in the urodynamic investigation of stress urinary incontinence. In patients with pelvic prolapse, urodynamic investigations should be performed during prolapse reposition.

Urodynamic study was available in Bangladesh at one or two private center before starting at BSMMU at 2012. Our center is now worked with quality service by experienced and dedicated personnel, as well as comprehensive report with further suggestions. From the beginning to now we are doing UDS with single operator. There was no review about the results and complications for UDS. Here is retrospective study of the UDS done in our center from beginning to till now.

\section{Materials and Methods}

This retrospective study was conducted at urology department of Bangabandhu Sheikh Mujib Medical University, Shahbag, Dhaka from January 2012 to April 2017. This is a tertiary health care center and urodynamic studies were conducted with a experienced persons. It has all the facilities to solve any health-related issues.

\section{Indications for urodynamic studies are:}

UDSs provide objective information on the occurrence of LUTSs and able to determination of a person's lower urinary tract (LUT) about normal or abnormal urinary tract physiology[6].

The most important target for treating LUT dysfunction is alleviating LUTSs. LUTSs may be related to a variety of pathophysiological processes and assessment on the basis of symptoms alone sometime leads to an incorrect diagnosis, specially in patients with a neurogenic bladder. It is therefore important to obtain objective data to make an accurate diagnosis. For example, there is only a weak association between the LPP and the severity of symptomatic urinary incontinence (UI) and a weak correlation between symptoms and results of UDSs (especially cystometry) in patients with UI $[5,8]$.

Uroflowmetry and measurement of post-voiding residual urine by ultrasonography are noninvasive tests that can be routinely performed in patients with LUTSs. The other UDSs are invasive tests and therefore should only be performed in selected cases. UDSs are indicated is the following circumstances: (1) when conservative treatment is ineffective; (2) when invasive treatment is being considered; (3) for protection of the upper urinary tract by creating a low-pressure reservoir and for controlling $\mathrm{UI}$; (4) for evaluation of the effects of treatment; and (5) for biofeedback training[9]. It is important for clinicians to evaluate the results of UDSs in relation to each patient's symptoms, to interpret the findings, data from the voiding diary, and results of clinical (or other) examinations[4].

\section{Urodynamic tests include}

- uroflowmetry

- postvoid residual measurement

- cystometric test

- leak point pressure measurement

- pressure flow study

- electromyography

- video urodynamic tests

\section{Standard operating procedure for urodynamics}

The UDS should be performed with aseptic precautions, counselling and verbal consent and according to the Good Urodynamics Practice Guidelines[10].

The equipment needed for the running of the urodynamic clinic include:

- catheter pack

- filling catheter

- abdominal and bladder pressure catheters

- Jasocaine jelly/sterile lubricant gel

- Four $\times 3$-way taps (depending on the type of transducers being used)

- Two $500 \mathrm{ml}$ bag of normal saline used for irrigation 
- One pump infusion set

- Three $\quad \times \quad$ pieces of tape (micropore to attach once catheters inserted; ensuring they stay in place during filling)

- One pair of sterile gloves

- non-sterile gloves

- paper roll to cover the couch

- towel or cover for the patient

- clean trolley with antiseptic wipes.

- If, for any reason, the test had to be abandoned, note this on the test form.

\section{Uroflowmetry (initial voiding test)}

- The patient is asked to attend clinic with a comfortably full bladder and bladder diary.

- The patient should be encouraged to sit in order to void into the voiding flow/volume transducer funnel mounted under the commode.

- The patient should be instructed to dispose of any tissues/wipes into the bin/bag provided and not into the flow metre.

- The utmost privacy must be maintained during the test and the patient should be made to feel comfortable and relaxed, enabling a usual voided pattern to be established.

- The maximum void flow rate and volume should then be recorded.

- The PVR volume should then be recorded using a drainage catheter and measuring container.

\section{Filing cystometry (catheterisation)}

It is essential that the machine is calibrated, set at zero at atmospheric pressure and a reference level for pressures should be established.

- Ideally the patient should be in the sitting position for the test. If this is not possible it should be recorded on the test form. A sheet should be provided for covering, maintaining dignity.

- Under aseptic technique, introduce catheters up through the urethra into the bladder and one into the rectum.

- Prior to filling, ask the patient to cough so that the traces can be observed. The spikes on the intravesical and intra-abdominal lines should be identical. Any necessary adjustments should be made and the cough repeated.
- Fill rate should be recorded on the test form, but is recommended as $100 \mathrm{ml}$ per minute.

- Ask the patient to cough every minute to ensure continued subtraction. If the lines slip, then stop the filling and rectify the problem.

- Complete test form with the number of millilitres at which the patient reports first, normal and strong desire to urinate, pain and volume leaked (if applicable).

- Total volume in the bladder at end of filling should be recorded.

- Detail any rise in detrusor pressure with or without urgency

Provocation test (while bladder is still filled)

While the intravesical and intra-abdominal lines are in situ, the patient should stand up on the incontinence sheet provided and the provocation tests such as running taps, coughing, etc., should be performed.

- Complete methods used and observations on the test form.

\section{Voiding cystometry}

- Allow patient to void into commode, recording peak flow rate, maximum void pressure and residual volume with the pressure lines still in. During this voiding phase, the patient's dignity and privacy must be maintained and staff should leave the room if necessary.

- Ask the patient to cough pre-void and post void to ensure adequate subtraction.

On completion of the investigation, the results may be explained to the patient and fluid advice should be given. Record diagnosis.

\section{Advice for patients}

All patient who have undertaken the test should be advised to expect some dysuria for up to 72 hours, their fluid intake should be increased during this time. The occurrence of systemic symptoms, pyrexia and malaise should be advised as an indication to seek medical advice a contact number should be provided if problems occur.

\section{Observation and Result}

In our center, Urology department of Bangabandhu Sheikh Mujib Medical University, we perform about 403 case of urodynamic studies. Most of the patients were referred from urology outpatient department OPD. In addition to this patient from gynecology (OPD), paediatric nephrology, neurosurgery of BSMMU, National Institute 
of Kidney Disease and Urology, Dhaka Medical College, and some private centers. We are performing UDS from January 2012 to till now. We have experienced manpower and two UDS machine. For patient safety and infection free procedure we always cautious and use new catheter. We do UDS with prior appointment with taking proper history, counselling of the patients, and brief discussion of the procedure. A bladder diary form discussed and essential material list handed to patient with proper contact address of us if need any further help. In case of continuous catheterised patient are changed to Clean Intermittent Self catheterization (CISC). Patient age range was 10-85 years. Female Patient was only 41. Among them most cases are equivocal and obstructive cases are ranked second about 68 cases $(16.87 \%)$, post operative cases sent for UDS was only six these were $\mathrm{BPH}$, stricture urethra, atonic bladder, and dysfunctional voiding. Impaired detrusor contractility is more common aetiology of bladder outlet obstruction but the final verdict again depends on the properly conducted pressure flow studies. The cause of underactive detrusor ranges from age to multipara, diabetes, and peripheral neuropathy. Only five patients had urinary tract infections (1.2\%) with rise of temperature but only two had cultural positive and treated with sensitive antibiotics. Organism were streptococcus aureus and pseudomonus. One female and one male patient develop retention and relived by continuous catheterisation and after 7 days removed catheter.

\section{Discussion}

According to the International Continence Society(ICS), LUT function is classified into detrusor and urethral function or filling and voiding function. Therefore, LUT dysfunction is classified as detrusor over-activity, an underactive detrusor, or an acontractile detrusor and the presence of an incompetent or obstructive urethra (Table 1)[1].

\section{Bladder outlet obstruction}

Male patients present with bladder neck obstruction, benign prostatic hyperplasia, or urethral stricture and female patients with pelvic organ prolapse or bladder neck obstruction may have bladder outlet obstruction (BOO). During the voiding phase, BOO may manifest as a high detrusor pressure with decreased urinary flow (obstructed pattern $1 / 4$ high pressure/low flow pattern) on an ICS nomogram or Schäfer nomogram obtained by pressure-flow studies. 2 The BOO index, formerly known as the Abrams-Griffith number $(1 / 4 \mathrm{PdetQmax}$ $2 \mathrm{Qmax}$ ), is $>40$ in patients with $\mathrm{BOO}$. During the filling phase, detrusor over-activity may be detected in 40$60 \%$ of patients with benign prostatic hyperplasia[11].

\section{Patients with Urinary Incontinence (UI)}

There are three main types of UI: urge UI, stress $\mathrm{UI}$ and mixed UI. Urgency UI is associated with overactive bladder ( $O A B$, also known as wet $O A B)$, and is considered to detrusor over-activity. Urodynamic stress $\mathrm{UI}$ is defined as the involuntary leakage of urine along with increased abdominal pressure in the absence of detrusor contraction during the filling phase. To assess urethral function during filling, the urethral pressure profilometry or (abdominal or detrusor) LPP is measured. UDSs should be performed in women prior to surgical intervention for stress UI. However, various studies have shown that the results of UDSs do not accurately predict the response to treatment of patients who have $O A B$ with or without detrusor over-activity, or in patients with urodynamic stress UI[12,13]. Practically, during UDS the association between symptoms of $O A B$ and detection of detrusor over-activity is weak, and currently it is not possible to confirm the response to treatment on the basis of quantification of detrusor over-activity during UDS ${ }^{14}$. There are conflict with results regarding the association between the severity of $\mathrm{UI}$ and urethral function tests. Measurements of urethral function (urethral pressure profilometry and LPP) are not useful for predicting the success after surgical treatment for stress UI. However, there is evidence that a lower suburethral sling procedure success rate with a low urethral (closing) pressure.

\section{Classification of Urinary tract dysfunction (ICS 2002)[1].}

\begin{tabular}{lll}
\hline & Filling phase & Voiding phase \\
\hline Detrusor function & DO & UAD, ACD \\
Bladder sensation & $\begin{array}{l}\text { Increased (Hypersensitive) } \\
\text { reduced (hyposensitive) }\end{array}$ \\
Incompetent & Obstructive \\
& & BOO \\
& & Dysfunctional Voiding \\
& DSD \\
& & Non relaxing \\
\hline
\end{tabular}

$\mathrm{DO}=$ Detrusor Overactivity, UAD =Underactive Detrusor, $\mathrm{ACD}=$ Acontractile Detrusor, $\mathrm{BOO}=$ Bladder Outlet Obstruction, and DSD= Detrusor Sphincter Dyssynergia. 


\section{Spinal cord lesion}

Patients with lower lumbar spine lesions, the common urodynamic findings are, such as intervertebral disc prolapse and spinal canal stenosis, are underactive or acontractile detrusor, but in cases of lumbar disc prolapse detrusor over-activity is also found in $10 \%$ of patients and lumbar canal stenosis cases $29 \%$ of those In these patients, urgency, urge Ul and, in males, erectile dysfunction are usually observed in association with intermittent claudication[15]. Cauda equina syndrome include bilateral sciatica, low back pain, saddle anesthesia and acute urinary retention. It typically occurs with central lumbar disc prolapse (the incidence is $1-5 \%$ of all prolapsed lumbar discs). Clinically, there is perineal sensory loss, voluntary control of both the anal and urethral sphincters, and sexual dysfunction caused by damage to the S2_S4 roots. The detrusor is not denervated and sympathetic innervation of the bladder neck may be preserved. In our study of eight patients, all of them still demonstrated acontractile detrusor up to 6 years after undergoing emergency surgery within 48 hours. Bladder function was irreversibly lost despite spinal surgery, while urethral function showed better recovery in patients with acute retention because of central lumbar disc prolapse. However, postoperatively most of those patients could only empty their bladder by changing the voiding posture or straining[16].

We are performing UDS from January 2012 to till now. We have experienced manpower and two UDS machine. For patient safety and infection free procedure we always cautious and use new catheter. We do UDS with prior appointment with taking proper history, counselling of the patients, and brief discussion of the procedure. A bladder diary form discussed and essential material list handed to patient with proper contact address of us if need any further help. In case of continuous catheterised patient are changed to Clean Intermittent Self catheterization (CISC). Patient age range was 10-85 years. Female Patient was only 41. Among them most cases are equivocal and obstructive cases are ranked second about 68 cases, post operative cases sent for UDS was only six these were BPH, stricture urethra, atonic bladder, and dysfunctional voiding. Impaired detrusor contractility is more common aetiology of bladder outlet obstruction but the final verdict again depends on the properly conducted pressure flow studies. The cause of underactive detrusor ranges from age to multipara, diabetes, and peripheral neuropathy. Only five patients had urinary tract infections with rise of temperature but only to had cultural positive and treated with sensitive antibiotics. Organism were streptococcus aureus and pseudomonus. One female and one male patient develop retention and relived by continuous catheterisation and after 7 days removed catheter. Treatment of these cases are not well documented and UDS report sent to patient to with request to handed to respective physicians.

\section{Conclusions}

Characteristic features of various types of lower urinary tract symptoms, including frequency, urgency, urinary incontinence, bladder outlet obstruction and neurogenic bladder, can be identified by UDSs. Video urodynamics is gold standard. Ambulatory urodynamics are very helpful in these regards. We are not yet in using video urodynamics instead urethrocystoscopy may help in assessing the urethra and bladder neck.

\section{References}

1. Yamanishi, T., Sakakibara, R., Uchiyama, T., \& Hirata, K. (2011). Role of urodynamic studies in the diagnosis and treatment of lower urinary tract symptoms. Urological Science, 22(3), 120-128. doi: 10.1016/j.urols.2011.08.007

2. Abrams PH, Blaivas JG, Stanton SL, Andersen JT. The standardisation of terminology of lower urinary tract function. The International Continence Society Committee on Standardisation of Terminology. Scand J Urol Nephrol 1988; 114(Suppl.):5-19.

3. Abrams P, Cardozo L, Fall M, Griffiths D, Rosier $\mathrm{P}$, Ulmsten U, et al; Standardisation Subcommittee of the International Continence Society. The standardisation of terminology of lower urinary tract function: report from the Standardisation Subcommittee of the International Continence Society. Neurourol Urodyn 2002; 21:167-78.

4. Rosier PF, Gajewski JB, Sand PK, Szabó L, Capewell A, Hosker GL; International Consultation on Incontinence 2008 Committee on Dynamic Testing. Executive summary: The International Consultation on Incontinence 2008 Committee on: "Dynamic Testing"; for urinary incontinence and for fecal incontinence. Part 1: Innovations in urodynamic techniques and urodynamic testing for signs and symptoms of urinary incontinence in female patients. Neurourol Urodyn 2010; 29: $140-5$.

5. Rosier, P. F., Giarenis, I., Valentini, F. A., Wein, A., \& Cardozo, L. (2014). Do patients with 
symptoms and signs of lower urinary tract dysfunction need a urodynamic diagnosis? ICI-RS 2013. Neurourology and Urodynamics, 33(5), 581586. doi:10.1002/nau.22580

6. Schäfer W, Abrams P, Liao L, Mattiasson A, Pesce F, Spangberg A, et al; International Continence Society. Good urodynamic practices: uroflowmetry, filling cystometry, and pressure-flow studies. Neurourol Urodyn 2002; 21:261-74.

7. Lowenstein L, Dooley Y, Kenton K, Rickey L, FitzGerald MP, Mueller E, et al. The volume at which women leak first on urodynamic testing is not associated with quality of life, measures of urethral integrity or surgical failure. J Urol 2007; 178:193-6.

8. Albo M, Wruck L, Baker J, Brubaker L, Chai T, Dandreo $\mathrm{KJ}$, et al. The relationships among measures of incontinence severity in women undergoing surgery for stress urinary incontinence. J Urol 2007; 177:1810-4.

9. Yamanishi T, Yasuda K, Murayama N, Sakakibara $\mathrm{R}$, Uchiyama $\mathrm{T}$, Ito $\mathrm{H}$. Biofeedback training for detrusor overactivity in children. J Urol 2000;164: $1686-90$.

10. Rachaneni, S., Mccooty, S., Middleton, L. J., Parker, V. L., Daniels, J. P., Coomarasamy, A., Latthe, P. (2016). Bladder ultrasonography for diagnosing detrusor overactivity: test accuracy study and economic evaluation. Health Technology Assessment, 20(7), 1-150. doi:10.3310/hta20070
11. Yamanishi T, Mizuno T, Tatsumiya $\mathrm{K}$, Watanabe M, Kamai T, Yoshida K. Urodynamic effects of silodosin, a new a1A-adrenoceptor selective antagonist, for the treatment of benign prostatic hyperplasia. Neurourol Urodyn 2010;29: 558-62.

12. Colli E, Artibani W, Goka J, Parazzini F, Wein AJ. Are urodynamic tests useful tools for the initial conservative management of non-neurogenic urinary incontinence? A review of the literature. Eur Urol 2003; 43:63-9.

13. Van Brummen $\mathrm{HJ}$, Heintz AP, van der Vaart $\mathrm{CH}$. The association between overactive bladder symptoms and objective parameters from bladder diary and filling cystometry. Neurourol Urodyn 2004; 23:38-42.

14. Malone-Lee J, Henshaw DJ, Cummings K. Urodynamic verification of an overactive bladder is not a prerequisite for antimuscarinic treatment response. BJU Int 2003; 92:415-7

15. Yamanishi T, Yasuda K, Sakakibara R, Murayama $\mathrm{N}$, Hattori T, Ito H. Detrusor overactivity and penile erection in patients with lower lumbar spine lesions. Eur Urol 1998; 34:360-4.

16. Y amanishi T, Yasuda K, Yuki T, Sakakibara R, Uchiyama T, Kamai T, et al. Urodynamic evaluation of surgical outcome in patients with urinary retention due to central lumbar disc prolapse. Neurourol Urodyn 2003; 22:670-5. 